\title{
Postoperative Radiotherapy for Endometrial Cancer in Elderly ( $\geq 80$ Years) Patients: Oncologic Outcomes, Toxicity, and Validation of Prognostic Scores
}

\author{
Eva Meixner 1,2,3,*iD, Kristin Lang 1,2,3, Laila König 1,2,3, Elisabetta Sandrini 1,2,3, Jonathan W. Lischalk 4 , \\ Jürgen Debus ${ }^{1,2,3,5,6}$ and Juliane Hörner-Rieber 1,2,3,6
}

1 Department of Radiation Oncology, Heidelberg University Hospital, Im Neuenheimer Feld 400, 69120 Heidelberg, Germany; kristin.lang@med.uni-heidelberg.de (K.L.); laila.koenig@med.uni-heidelberg.de (L.K.); elisabetta.sandrini@med.uni-heidelberg.de (E.S.); juergen.debus@med.uni-heidelberg.de (J.D.); juliane.hoerner-rieber@med.uni-heidelberg.de (J.H.-R.)

2 Heidelberg Institute of Radiation Oncology (HIRO), Im Neuenheimer Feld 400, 69120 Heidelberg, Germany

3 National Center for Tumor Diseases (NCT), Im Neuenheimer Feld 460, 69120 Heidelberg, Germany

4 Perlmutter Cancer Center, Department of Radiation Oncology, New York University Langone Health, New York, NY 10023, USA; jonathan.lischalk@nyulangone.org

5 Heidelberg Ion Therapy Center (HIT), Im Neuenheimer Feld 450, 69120 Heidelberg, Germany

6 German Cancer Research Center (DKFZ), Clinical Cooperation Unit Radiation Oncology,

check for updates

Citation: Meixner, E.; Lang, K. König, L.; Sandrini, E.; Lischalk, J.W.; Debus, J.; Hörner-Rieber, J. Postoperative Radiotherapy for Endometrial Cancer in Elderly ( $\geq 80$ Years) Patients: Oncologic Outcomes, Toxicity, and Validation of Prognostic Scores. Cancers 2021, 13, 6264. https://doi.org/10.3390/ cancers13246264

Academic Editors: Elena Paillaud, Marije E. Hamaker and Pierre Soubeyran

Received: 21 November 2021 Accepted: 12 December 2021 Published: 14 December 2021

Publisher's Note: MDPI stays neutral with regard to jurisdictional claims in published maps and institutional affiliations.

Copyright: (c) 2021 by the authors. Licensee MDPI, Basel, Switzerland. This article is an open access article distributed under the terms and conditions of the Creative Commons Attribution (CC BY) license (https:// creativecommons.org/licenses/by/ $4.0 /)$. Im Neuenheimer Feld 280, 69120 Heidelberg, Germany

* Correspondence: Eva.Meixner@med.uni-heidelberg.de

Simple Summary: As population ages, understanding of frailty in cancer patients becomes all the more important. Due to the rarity of elderly patients in randomized prospective trials, only limited data exist regarding safety and feasibility of postoperative radiotherapy for very elderly women of 80 years or older in a curative treatment intent. Further, geriatric assessments and prognostic scores for these women are not sufficiently validated. In a homogenous cohort of very elderly women with endometrial cancer, we confirmed that, despite older age, adjuvant radiotherapy can achieve excellent local control and overall survival with minimal high-grade toxicity. The geriatric G8 screening score was a highly applicable tool for prognostic evaluation of overall survival in our review.

Abstract: Endometrial cancer is a common malignancy in elderly women that are more likely to suffer from limiting medical comorbidities. Given this narrower therapeutic ratio, we aimed to assess the oncologic outcomes and toxicity in the adjuvant setting. Out of a cohort of 975 women, seventy patients aged $\geq 80$ years, treated with curative postoperative radiotherapy (RT) for endometrial cancer between 2005 and 2021, were identified. Outcomes were assessed using Kaplan-Meier-analysis and comorbidities using the Charlson Comorbidity Index and G8 geriatric score. The overall survival at 1-, 2- and 5-years was $94.4 \%, 82.6 \%$, and $67.6 \%$, respectively, with significant correlation to G8 score. At 1- and 5-years, the local control rates were $89.5 \%$ and $89.5 \%$ and distant control rates were $86.3 \%$ and $66.9 \%$, respectively. Severe ( $\geq$ grade 3 ) acute toxicity was rare with gastrointestinal $(2.9 \%)$, genitourinary $(1.4 \%)$, and vaginal disorders $(1.4 \%)$. Univariate analysis significantly revealed inferior overall survival with lower RT dose, G8 score, hemoglobin levels and obesity, while higher grading, lymphangiosis, RT dose decrease and the omission of chemotherapy reduced distant control. Despite older age and additional comorbidities, elderly patients tolerated curative treatment well. The vast majority completed treatment as planned with very low rates of acute severe side-effects. RT offers durable local control; however, late distant failure remains an issue.

Keywords: geriatric care; vulnerability; Charlson Comorbidity Index (CCI); G8 geriatric screening score; uterine neoplasm; high-dose-rate brachytherapy; adjuvant therapy 


\section{Introduction}

Endometrial cancer (EC) represents the fourth most common cancer in women in the United States with a rising incidence and a mean age at diagnosis of 68 years [1,2]. Known risk factors for EC development include body mass index (BMI) and estrogen therapy [3-5]. The most common clinical presentation is postmenopausal bleeding which is typically evaluated by transvaginal ultrasound and subsequent endometrial sampling [6,7]. Pathological classification originally emerged from two classical phenotypes and includes Type I which is closely linked to aforementioned metabolic components, and Type 2 representing a higher risk cohort with poor differentiation and inferior clinical outcome [8]. Correlations of these two types with histologic patterns have been defined with the most common variants being endometrioid adenocarcinoma, serous and clear-cell carcinoma, and carcinosarcoma $[9,10]$. Staging follows the classification of the International Federation of Gynecology and Obstetrics (FIGO) Cancer Report [11] and corresponding TNM classification.

Radical hysterectomy and bilateral salpingo-oophorectomy represent the cornerstone of curative therapy [11,12]. Ongoing discussions remain regarding the necessity and extent of surgical lymph node assessment and sentinel lymph node mapping in this disease site [13]. Adjuvant oncological management consists of a broad variety of radiotherapeutic and often times chemotherapy options. Risk-stratified recommendations are constantly updated, particularly with respect to the results of the Cancer Genome Atlas [14] and ESMOESGO-ESTRO consensus [15] which aim for a personalized molecular-based treatment approach. From a radiation standpoint, external beam radiotherapy (EBRT) is utilized in cases of locally advanced tumors or localized disease with demonstrated high risk factors. Adjuvant radiotherapy (RT) has been proven to significantly reduce locoregional relapse from $15.5 \%$ to $6 \%$, as observed in the 15-year long-term PORTEC1-analysis [16]. Similarly, vaginal brachytherapy (BT) has been shown to achieve a comparable outcome to EBRT in PORTEC2-trial [17] for intermediate risk EC with concordant improvements in gastrointestinal toxicity. Ultimately, 5-year overall survival (OS) rates have been reported to be 81 to $96 \%$ for early-stages with a 2-year risk of local relapse at 3\% following RT for intermediate-risk patients $[16,18,19]$.

In the modern era, the use of patient-tailored adjuvant therapy aims to optimize oncologic outcome, however, similar to many published studies, it is often reserved for women with good clinical performance status. Elderly patients with multiple medical comorbidities may have worsened performance status, thus narrowing the therapeutic window for adjuvant treatment. Oncologic care for this patient population requires multidisciplinary management in effort to offer curative therapy while reducing toxicity and preserving quality of life. Nevertheless, despite EC being a disease of elderly women, oncogeriatric patients represent a minority of the patient population included in published studies [20-22]. As such, detailed research in this patient population is required to better characterize cancer and toxicity outcomes.

Comprehensive geriatric and comorbidity assessments currently exist to evaluate the oncogeriatric patient and include the Adult Comorbidity Evaluation 27 (ACE-27) [23], Charlson Comorbidity Index (CCI) [24], and G8 screening score [25]. Such assessments offer a standardized way to characterize the risk and feasibility of offering aggressive cancer treatment in this patient population. Unfortunately, most of these scales are tailored for perioperative mortality and surgical outcomes rather than RT $[20,26]$. Although, in the acute setting, RT can be much less toxic than surgery, the logistical burden of extended EBRT can be challenging and late toxicity requires better categorization. Ultimately, EC treatment for elderly patients is complex and consists of highly individual choices for which feasibility of optimal treatment must be evaluated.

Therefore, the aim of the current study is to evaluate oncologic outcome and treatmentrelated acute toxicity of postoperative curative RT in elderly patients diagnosed with EC, as well as analyze prognostic factors for improved identification of vulnerable patients. 


\section{Materials and Methods}

\subsection{Patient and Treatment Characteristics}

Out of a sample of 975 women, who were treated with curative, postoperative RT following surgical resection for EC between March 2005 and June 2021 at our Department of Radiation Oncology of Heidelberg University Hospital, we included a subset of women aged $\geq 80$ years. Primary focus was set by a threshold of 80 years due to the underrepresentation of this subgroup in prior and ongoing analyses and considering the growing elderly cancer population. The single-institutional retrospective analysis was approved by the local ethics committee (S-453/2021). Patient and oncologic data were individually assessed for each woman and included the following: Demographic, comorbidity, clinical gynecological exam, transvaginal ultrasound, computed tomography, magnetic resonance imaging and cysto- and rectoscopy, if available. Each patient was carefully classified according to FIGO staging system [11].

\subsection{Radiotherapy and Oncological Treatment}

Treatment recommendations were documented according to current national guidelines and patients' individual status as discussed in an interdisciplinary tumor conference with input from gynecological oncology, radiation oncology, radiology, medical oncology, and pathology. All patients underwent upfront radical hysterectomy at a minimum, and were subsequently treated with adjuvant RT with curative intent. Stage-adapted surgical treatment was performed according to oncologic recommendations with the exception of cases where comorbidities precluded optimal surgical treatment. Of note, pelvic lymph node dissection was performed according to local institutional guideline recommendations, though in some cases, due to patient performance status or preference, this was deferred.

Radiotherapy was delivered in an adjuvant setting utilizing BT alone or BT in combination with EBRT. Radiotherapy modality was based on FIGO staging, although in some cases, due to poor performance status or patient refusal, EBRT was deferred. EBRT was delivered utilizing a 6-23 MV linear accelerator either as 3-dimensional conformal radiotherapy (3D-CRT) or intensity modulated radiation therapy (IMRT). Contouring of the clinical target volume (CTV) for EBRT was performed according to the updated institutional guidelines $[27,28]$ and each patients' individual risk for the pelvic region including the upper vagina, vaginal cuff, paravaginal and parametrial tissues. The nodal CTV further encompassed the following regions: the bifurcation of the aorta and presacral area as well as the common iliac, obturator and external and internal iliac. A margin of 0.5 to $1.5 \mathrm{~cm}$ was applied for the planning target volume (PTV) depending on the RT technique and organ motions. For EBRT, conventional dose fractionation was utilized delivered on a once daily schedule. Dose constraints for adjacent organs at risk (OARs) were set according to the Quantec recommendations [29]. All patients received a vaginal cuff high-dose-rate (HDR) BT, using Iridium-192 with intracavitary single or multichannel applicator according to American Brachytherapy Society Consensus Guidelines [30]. For further comparison, an equivalent dose in 2 Gy fractions (EQD2) was calculated for EBRT and HDR BT using the linear-quadratic model (1):

$$
\begin{aligned}
& \text { EQD2Gy = fractional dose } \times \text { number of fractions } \times(\text { fractional dose }+ \\
& \qquad \alpha / \beta) /(2 \mathrm{~Gy}+\alpha / \beta)
\end{aligned}
$$

Tumor dose summations were calculated with an $\alpha / \beta$ ratio of 10 assumed for the tumor.

\subsection{Toxicity, Oncologic Follow-Up, and Prognostic Factors}

Toxicity was classified according to Common Terminology Criteria for Adverse Events (CTCAE, version 5.0). Any new onset of symptoms within the start of RT up to 90 days was considered as acute toxicity, late-toxicity was defined by a time interval of $>90$ days during the follow-up period and assessed at first clinical examination after radiotherapy. Clinical oncologic outcome included the analysis of OS, local control (LC), and distant control (DC) 
and was defined as the time interval from the start of RT until death from any cause or at last contact, and until the first occurrence of local or distant recurrence. Local recurrence was considered as any tumor progression at the primary site or regional pelvic lymph nodes. DC was specified as development of metastatic lesions occurring outside the pelvis. For evaluation of treatment response, follow-up included any medical information from clinical examinations, referring physician notes and radiology.

For comorbidity and geriatric assessments, CCI [24] and G8 screening score [25] have been proven to have a high sensitivity in studies with cancer patients and prediction of ageadjusted comorbidities for estimation of 10-year survival rates [31]. Required factors, such as nutrition status, cognitive and depression scale, clinical performance, and comorbidities, were evaluated. Due to the retrospective nature of the study, reliable full detail data for G8 scoring were only available for a subset of women.

\subsection{Statistical Analysis}

Kaplan-Meier analysis and the log-rank test or Cox regression were utilized for the calculation of OS, LC, and DC during the follow-up period and further to compare survival curves and subgroups. A $p$-value of less than 0.05 was considered as statistically significant. Uni- and multivariate log-rank test and Cox-proportional hazard ratios (HR) with a 95\% Confidence Interval (CI) were applied to analyze the influence of prognostic factors. Only significant variables from the univariate analysis were included to multivariate Cox models. Statistical analysis was performed with the statistical software IBM SPSS statistics (versions 25 and 28, Armonk, NY, USA).

\section{Results}

\subsection{Patient and Tumor Characteristics}

Out of 975 women treated with postoperative radiotherapy for EC, a total of 70 patients (7.2\%) with a median age of 82 years (range: 80-95 years) were identified that met our inclusion criteria. From a performance status standpoint, the median Karnofsky score was 80 (60-100) with a median Charlson comorbidity index of 5 (4-9). The median G8 screening score was found to be 13 (6-16) and was assessed in a subset of 58 patients who had complete information available for analysis. The vast majority of patients in the overall cohort ( $\geq 80$ years) and in the subgroup of patients, for whom the G8 screening was available, were diagnosed with FIGO stage I or II disease (overall cohort: $n=58,82.9 \%$; subgroup: $n=48,82.8 \%$ ). The predominant histologic subtype was endometrioid ( $n=51$, $72.9 \%$ ), which is consistent with epidemiological data. For the definition of the histologic grading, serous carcinoma and carcinosarcoma were considered to be grading G3. Patient characteristics are detailed in Table 1.

\subsection{Treatment Characteristics}

Overall, the majority of patients underwent an oncologic surgery ( $n=62,88.6 \%$ ) with the remaining patients undergoing a non-oncologic excision due to patient comorbidities or preference. There was a wide variety of lymph node assessment in our cohort with a relatively even distribution of patients not requiring a lymph node dissection, undergoing a lymph node dissection, or deferring a recommended dissection due to toxicity risk or patient preference. Of note, very few patients underwent adjuvant chemotherapy $(n=1,1.43 \%)$ despite an indication for systemic therapy in over half of our cohort. This is a reflection of the limited performance status and median age of our patient population.

Postoperative radiation therapy was performed as indicated in the plurality of patients $(n=57,81.4 \%$ ). The median time from surgery to the start of radiation was 54 days with a median radiation treatment length of 25 days. Brachytherapy alone was performed in the majority of cases $(n=36,51.4 \%)$, with the remaining receiving a combination of EBRT and BT. EBRT type was evenly distributed between IMRT $(n=16,22.9 \%)$ and 3D-CRT ( $n=18$, $25.7 \%$ ). For EBRT, a median dose of $45.0 \mathrm{~Gy}(19.8-56 \mathrm{~Gy})$ in 11 to 28 once-daily fractions was employed. For women treated with BT only, a median dose of 22.0 Gy (5-22 Gy) in 1 
to 4 fractions was delivered. In those who received BT as boost in combination with EBRT, a median boost dose of $10 \mathrm{~Gy}(10-25 \mathrm{~Gy})$ in 2 to 5 fractions was used. Four patients (5.7\%) did not complete radiation treatment as planned, three due to grade 3 acute toxicity, one due to refusal of any further adjuvant therapy. Treatment characteristics are detailed in Tables 1 and 2 .

Table 1. Patient characteristics.

\begin{tabular}{|c|c|}
\hline Characteristics & Values \\
\hline Median age & 82 (range: $80-95$ ) years \\
\hline Median Karnofsky performance score & $80(60-100) \%$ \\
\hline Median Charlson Comorbidity Index & $5(4-9)$ \\
\hline Median G8 screening score & $13(6-16) *$ \\
\hline Median BMI & $26.5(20.5-45.7)$ \\
\hline Median hemoglobin level & $11.9(8.9-14.0) \mathrm{g} / \mathrm{dL}$ \\
\hline \multicolumn{2}{|l|}{ FIGO stage } \\
\hline $1 / 2$ & $58(82.9 \%)$ \\
\hline $3 / 4$ & $12(17.1 \%)$ \\
\hline \multicolumn{2}{|l|}{ Surgical procedure } \\
\hline oncological procedure & $62(88.6 \%)$ \\
\hline non-oncologic excision only & $8(11.4 \%)$ \\
\hline \multicolumn{2}{|l|}{ Adjuvant chemotherapy } \\
\hline not indicated & $30(42.9 \%)$ \\
\hline performed & $1(1.43 \%)$ \\
\hline indicated, but not feasible & $39(55.7 \%)$ \\
\hline \multicolumn{2}{|l|}{ Lymph node dissection } \\
\hline not indicated & $20(28.6 \%)$ \\
\hline performed & $25(35.7 \%)$ \\
\hline indicated, but not feasible & $25(35.7 \%)$ \\
\hline \multicolumn{2}{|l|}{ Postoperative radiotherapy } \\
\hline performed as indicated & $57(81.4 \%)$ \\
\hline EBRT indicated, but not feasible & $13(18.6 \%)$ \\
\hline \multicolumn{2}{|l|}{ Histological subtype } \\
\hline endometrioid & $51(72.9 \%)$ \\
\hline serous & $13(18.6 \%)$ \\
\hline carcinosarcoma & $6(8.6 \%)$ \\
\hline
\end{tabular}

Table 2. Treatment characteristics.

\begin{tabular}{cc}
\hline Characteristics & Values \\
\hline Median time from surgery to start of RT & 54 (range: $6-219)$ days \\
Median treatment time & $25(1-67)$ days \\
Median days of RT discontinuation & $0(0-5)$ days \\
Premature cessation of RT & $4(5.7 \%)$ \\
Median total dose in EQD2 $(\alpha / \beta=10)$ & $28.42(6.25-77.98)$ Gy \\
RT technique & $16(22.9 \%)$ \\
IMRT (EBRT + BT) & $18(25.7 \%)$ \\
3D-CRT (EBRT + BT) & $36(51.4 \%)$ \\
BT only &
\end{tabular}

3D-CRT: three-dimensional conformal radiotherapy, BT: brachytherapy, EQD2: equivalent dose in 2 Gy fractions, IMRT: intensity-modulated radiotherapy, RT: radiotherapy.

\subsection{Toxicity}

The most common low-grade ( $\leq$ grade 2$)$ acute toxicity observed was as follows: gastrointestinal $(n=31,44.3 \%)$, genitourinary $(n=25,35.7 \%)$, vaginal mucositis $(n=17$, $24.3 \%)$, and fatigue $(n=10,14.3 \%)$ and significantly more often observed in patients with EBRT plus BT compared to BT alone $(p=0.013)$. Gastrointestinal toxicity ( $\leq$ grade 2$)$ in 31 patients included one of the following or a combination of diarrhea $(n=20)$, nausea 
$(n=6)$, abdominal pain $(n=5)$, and obstipation $(n=3)$, while genitourinary disorders were mainly dysuria $(n=14)$, urinary frequency/urgency $(n=12)$, incontinence $(n=6)$, and nycturia $(n=2)$. Extended para-aortic field RT was applied only once $(n=1,81$ years) due to more than twenty pathologically confirmed metastatic para-aortic lymph nodes, leading to grade 2 gastrointestinal toxicity without hospitalization but RT discontinuation for two days.

Overall, severe ( $\geq$ grade 3 ) acute toxicity was rare and included the following rates: gastrointestinal $(2.9 \%)$, genitourinary $(1.4 \%)$, and vaginal disorders $(1.4 \%)$. Higher grade acute toxicity occurred in two patients with grade 3 gastrointestinal symptoms $(n=2,2.9 \%)$ of severe diarrhea with electrolyte abnormalities and one woman with grade 3 vaginal cuff dehiscence $(n=1,1.4 \%)$ who had insufficient wound healing post hysterectomy. In each case of high-grade toxicity, this led to cessation of RT treatment early. One woman (1.4\%) with urinary disorders and a grade 3 infection required intravenous antibiotics leading to urinary retention ultimately finished therapy without discontinuation with supportive care. Therapy interruption of one to five days was documented for 23 (32.9\%) patients, leading to a premature termination of RT for four (5.7\%) women. Inpatient treatment was necessary for 28 patients $(40.0 \%)$ with a median duration of four (2-48) days. Median weight loss during RT was $0(-10$ to +7$)$ kilogram.

Concerning late-toxicity, fatigue ( $\leq$ grade 2 ) was the most common side effect, occurring in nine patients $(12.9 \%)$. Only two patients experienced a new onset of gastrointestinal toxicity $(2.9 \%)$ in the form of low-grade ( $\leq$ grade 2$)$ obstipation $(n=1)$ and nausea plus diarrhea $(n=1)$, while three patients suffered from new and increasing genitourinary $(4.3 \%)$ side effects with worsening incontinence $(n=2)$ and new dysuria $(n=1)$.

\subsection{Oncologic Outcomes}

Oncologic outcomes for the overall group of 70 patients with a median follow-up time of 4.9 months (0-128.0 months) is shown in Table 3. Kaplan-Meier curves for oncologic outcomes are displayed in Figure 1. The 1-, 2- and 5-year OS rates were 94.4\%, 82.6\%, und $67.6 \%$, respectively (Figure 1A). Local failures were detected in three women (4.3\%) within the first year after RT, with a median time to relapse of 9.6 months (4.5-10.2 months). This resulted in 1-, 2-, and 5-year LC rates of each 89.5\% (Figure 1B). During the follow-up period, a total of nine patients were diagnosed with distant metastases after a median of 12.2 months following RT (2.6-49.3) and with the following organ distribution: peritoneal $(n=4)$, lymphatic outside pelvis $(n=4)$, pulmonary $(n=2)$, bone $(n=2)$, hepatic $(n=1)$, spleen $(n=1)$, brain $(n=1)$, skin $(n=1)$, pleural $(n=1)$ lesions. DC rates were of $86.3 \%$, $73.6 \%$ and $66.9 \%$ (Figure $1 C$ ) after 1 -, 2 - and 5-years, respectively.

Table 3. Outcome with Kaplan-Meier estimates.

\begin{tabular}{cccc}
\hline Rates & Overall Survival & Local Control & Distant Control \\
\hline 1-year & $94.4 \%$ & $89.5 \%$ & $86.3 \%$ \\
2-year & $82.6 \%$ & $89.5 \%$ & $73.6 \%$ \\
5-year & $67.6 \%$ & $89.5 \%$ & $66.9 \%$ \\
\hline
\end{tabular}

\subsection{Prognostic Factor Analysis}

Univariate analysis of prognostic factors influencing oncologic outcomes was performed in detail (Table 4). None of the assessed clinical or tumor-related factors were found to be associated with local control outcome. Univariate analysis revealed lower histologic tumor grading (Hazard Ratio (HR) 34.098, (Confidence Interval (CI): 0.82-1411.8), $p=0.001$ ) and absence of lymph vascular involvement (HR 5.581 (CI: 0.979-31.829), $p=0.031$ ) were significantly associated with lower rates of distant metastases. Additionally, treatment with an EQD2 below 60 Gy (HR 8.969 (CI: 1.766-45.543), $p=0.008$ ) and the omission of adjuvant chemotherapy was highly associated with inferior DC (HR 2.236 (CI: 0.925-5.405), $p<0.0001)$. Multivariate analysis could not identify any statistically significant associations for either LC or DC. 


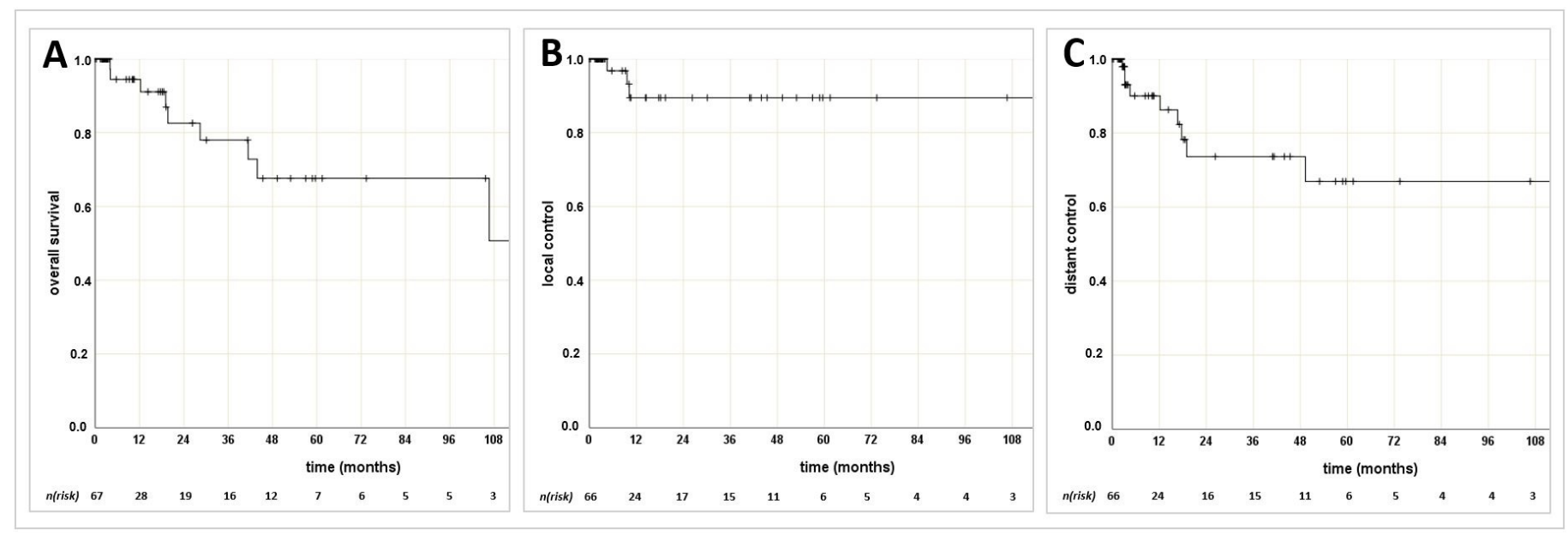

Figure 1. Kaplan-Meier curves for (A) overall survival, (B) local control, (C) distant control, $n$ (risk): number at risk.

Table 4. Univariate analyses.

\begin{tabular}{|c|c|c|c|c|c|c|}
\hline \multirow[t]{2}{*}{ Characteristics } & \multicolumn{2}{|c|}{ Overall Survival } & \multicolumn{2}{|c|}{ Local Control } & \multicolumn{2}{|c|}{ Distant Control } \\
\hline & HR 95\%CI & $p$ & HR 95\%CI & $p$ & HR 95\%CI & $p$ \\
\hline Age & $0.937(0.732-1.200)$ & 0.608 & $1.005(0.734-1.375)$ & 0.976 & $0.668(0.440-1.015)$ & 0.059 \\
\hline $\begin{array}{l}\text { Body mass index } \\
\text { BMI }<28 \text { vs. } \geq 28\end{array}$ & $1.102(0.989-1.228)$ & 0.033 & $1.112(0.778-1.589)$ & 0.208 & $0.936(0.696-1.259)$ & 0.137 \\
\hline Charlson Comorbidity Index & $1.197(0.706-2.030)$ & 0.505 & $1.401(0.648-3.026)$ & 0.391 & $0.657(0.335-1.288)$ & 0.221 \\
\hline G8 Screening Score & $0.666(0.503-0.883)$ & 0.005 & $1.438(0.700-2.955)$ & 0.323 & $0.936(0.666-1.317)$ & 0.705 \\
\hline $\begin{array}{c}\text { Karnofsky performance score } \\
\leq 80 \text { vs. } \geq 90\end{array}$ & $1.215(0.245-6.030)$ & 0.811 & $0.034(0-4223.770)$ & 0.572 & $3.049(0.816-11.397)$ & 0.081 \\
\hline Number of medications & $0.898(0.690-1.167)$ & 0.420 & $0.970(0.547-1.723)$ & 0.918 & $0.931(0.710-1.220)$ & 0.603 \\
\hline $\begin{array}{l}\text { Hemoglobin level } \\
\leq 12 \text { vs. }>12 \text { g/dL }\end{array}$ & $0.155(0.019-1.267)$ & 0.046 & $47.435(0-5,464,301)$ & 0.516 & $0.522(0.124-2.195)$ & 0.375 \\
\hline $\begin{array}{l}\text { Histologic grading } \\
\text { G1 vs. G2 vs. G3 }\end{array}$ & $2.412(0.749-7.767)$ & 0.140 & $1.274(0.260-6.256)$ & 0.765 & $34.098(0.82-1411.8)$ & 0.001 \\
\hline $\begin{array}{l}\text { FIGO stage } \\
1 / 2 \text { vs. } 3 / 4\end{array}$ & $0.993(0.122-8.089)$ & 0.995 & $0.042(0-335,213.6)$ & 0.695 & $1.881(0.388-9.122)$ & 0.433 \\
\hline $\begin{array}{c}\text { TNM } \\
\text { T1/2 vs. T3/4 }\end{array}$ & $34.49(2.158-551.6)$ & 0.012 & $0.031(0-1604.964)$ & 0.529 & $8.762(0.792-96.963)$ & 0.077 \\
\hline $\begin{array}{l}\text { Nodal stage } \\
\text { N0 vs. N+ }\end{array}$ & $0.993(0.122-8.089)$ & 0.995 & $0.042(0-335,213.6)$ & 0.695 & $2.110(0.434-10.251)$ & 0.354 \\
\hline $\begin{array}{l}\text { Tumor type } \\
\text { endometrioid vs. serous vs. } \\
\text { carcinosarcoma }\end{array}$ & $3.400(1.427-8.100)$ & 0.006 & $0.052(0-294.620)$ & 0.503 & $1.770(0.638-4.914)$ & 0.273 \\
\hline $\begin{array}{l}\text { Lymph vascular involvement } \\
\text { L1 vs. L0 }\end{array}$ & $0.470(0.055-4.033)$ & 0.491 & 1.149 (0.104-12.68) & 0.910 & $5.581(0.979-31.829)$ & 0.031 \\
\hline $\begin{array}{l}\mathrm{EQD} 2 \geq 60 \mathrm{~Gy}(\alpha / \beta=10) \\
\text { Omission of indicated therapy }\end{array}$ & $33.062(3.618-302)$ & 0.002 & $0.038(0-32,130.29)$ & 0.639 & $8.969(1.766-45.543)$ & 0.008 \\
\hline Oncological surgery & $0.043(0-2466.448)$ & 0.574 & $0.044(0-2,913,770)$ & 0.733 & $0.040(0-277.695)$ & 0.476 \\
\hline Lymph node dissection & $1.094(0.459-2.609)$ & 0.839 & $0.616(0.134-2.836)$ & 0.534 & $1.636(0.665-4.026)$ & 0.284 \\
\hline Chemotherapy & $1.122(0.572-2.202)$ & 0.738 & $0.733(0.221-2.435)$ & 0.613 & $2.236(0.925-5.405)$ & $<0.0001$ \\
\hline EBRT & $1.013(0.199-5.164)$ & 0.987 & $0.039(0-44,883.5)$ & 0.649 & $0.600(0.074-4.873)$ & 0.633 \\
\hline
\end{tabular}

BMI: body-mass index, EBRT: external beam radiotherapy, EQD2: equivalent dose in 2 Gy fractions, FIGO: International Federation of Obstetrics and Gynecology, CI: confidence interval, DC: distant control, HR: hazard ratio, LC: local control, OS: overall survival, RT: radiotherapy. For continuous variables, the HRs represents a relative increase in risk according to unit change. A $p$-value of $<0.05$ (bold in the table) was considered statistically significant.

The extent of the tumor according to the TNM classification was a reliable prognostic factor for inferior OS in locally advanced tumors (HR 34.490 (CI: 2.158-551.55), $p=0.012$ ). Furthermore, the histological tumor subtype was significantly associated with the outcome of OS (HR 3.400 (CI: 1.427-8.100), $p=0.006$ ) with endometroid adenocarcinoma being the most favorable and carcinosarcoma being the least favorable histologic subtype. A BMI of 28 or higher (HR 1.102 (CI: 0.989-1.228), $p=0.033$ ) and anemia with a hemoglobin level below 12 (HR 0.155 (CI: 0.019-1.267), $p=0.046$ ) were significantly associated with an inferior OS on univariate analysis. Utilization of an equivalent dose in 2-Gy fractions 
(EQD2 dose) for a $\alpha / \beta=10$ of at least $60 \mathrm{~Gy}$ showed superior OS outcome (HR 33.062 (CI: 3.618-302.1), $p=0.002$ ). For frailty assessment, the G8 rating (HR 0.666 (CI: 0.503-0.883), $p=0.005)$ was identified as a significant factor for OS prediction, whereas CCI score was not reliable for this patient cohort. Multivariate analysis was not significant for OS for any of the evaluated factors.

\section{Discussion}

Endometrial cancer is a common gynecologic malignancy of elderly women with a growing spectrum of individualized therapeutic approaches due to constantly updated molecular and genetic research. Elderly patients with multiple medical comorbidities represent a large cohort of patients treated in the clinic, but are often times underrepresented in published randomized trials [32]. Therapeutic recommendations based strictly on age stratification are insufficient in differentiating fit versus frail patients, particularly as life expectancy continues to improve. Theoretically, from a technical standpoint, there is almost no limit to aggressive radiotherapeutic approaches making patient selection in a multidisciplinary setting critical to determine a personalized treatment approach based not only on patient age but medical comorbidities, performance status, and patient preference.

Certainly, age alone has been described as a risk factor for inferior outcomes in the historical literature [33,34]. Alektiar et al. [34] reported 5-year OS of EC patients $<70$ years $(n=321)$ was significantly higher than in women age $\geq 70$ years $(n=84), 92 \%$ vs. $80 \%$ $(p=0.006)$, respectively. In fact, a comparable detriment in oncologic outcome was observed for 5-year LC rates in patients age greater than 70 years $(89 \%$ vs. 97\%, $p=0.02)$. A patient cohort of 53 women age $\geq 75$ years treated with postoperative RT for EC was analyzed by Fiorica et al. [35] and they found a 5-year OS of 72.3\% and a 5-year LC of $82.7 \%$. In the present study, a larger group of 70 women with different inclusion criteria, all aged 80 years or older, were analyzed and showed an estimated 5-year LC rate of $89.5 \%$, comparable to the aforementioned data. In contrast, our data showed an inferior 5-year OS rate of only $67.7 \%$, which is likely due to the comparable older age of the present cohort (median age of 82 years).

The omission of postoperative RT has been reported more often in older patients and is consistent with observed inferior LC [36]. Our study could not prove a significant correlation of outcome to the omission of indicated EBRT in a small number of 13 patients. However, despite the deferral of EBRT, BT alone was performed in this same subset. This was thought to be an optimal approach given the short overall treatment time and mitigation of treatment-related toxicity. This method has been previously reported in the literature to be a successful management strategy for women in poor general health [37].

Interestingly, the omission of chemotherapy (in warranted cases) led to an inferior distant control rate, which was notable in the oncologic outcomes of the present study. Indeed, in managing patients with a very narrow therapeutic window, the feasibility of toxic chemotherapy is restricted, which reflects the limited performance status and median age of our patient population. Thus, modification and risk-stratification for a less toxic systemic therapy regimes is a critical topic for future research [38].

Treatment-related toxicity is an important aspect of the analysis of any treatment regimen offered to high-risk patient population. Fiorica et al. [35] reported an elevated occurrence of low-grade gastrointestinal toxicities of $73.6 \%$, but without occurrence of any severe toxicity. In comparison, our results showed a less notable low-grade toxicity rate of $44.3 \%$, however, two patients developed grade 3 radiation-induced toxicity. Of note, no patients experienced any grade 4 or higher toxicity. Genitourinary, gastrointestinal, and vaginal side effects are well known in the treatment of gynecological cancers, but in our cohort, overall rates of severe toxicity were limited in these realms. Nonetheless, even in the presence of only so-defined 'low'-grade toxicity, the rate of unplanned hospitalization in our study was high (40.0\%) and prior studies of gastrointestinal cancer patients over 65 years have shown even higher rates up to 53\% for a different RT region [39]. This is most likely reflecting the overall higher risk of therapy-induced impairment of quality 
of life and physical or psychological status in the very elderly due to frailty and clinical comorbidities [40].

It is worth pointing out that fatigue was a common toxicity observed by our elderly cohort. As described by Giacalone et al. [41], radiation-related fatigue can be multifactorial in nature, particularly in the elderly. Of note, our study also showed poorer overall survival in the presence of anemia, a common cause of fatigue. Thus, especially in older patients, special focus should be placed on the management of fatigue during RT. Duska et al. [21] advocate for a comprehensive offer of standard treatment approaches also to the elderly, but with thoughtful consideration and implementation of toxicity-sparing techniques, such as image-guided IMRT has proven in the treatment of pelvic gynecological malignancies [42]. Furthermore, from a radiation oncologist view, there has been great RT technique development over the assessed time of the last 15 years. The application of the standard technique has today changed from 3D-RT into widespread advanced IMRT, that has been proven to improve bone marrow sparing and more conformal target coverage and thus to be associated with lower gastrointestinal and hematologic toxicity than 3D-RT without diminishing the oncologic outcome [43]. The use of above-mentioned BT or the application of smaller pelvic RT volumes can be options to spare toxicity [44] and are even more attractive in the treatment of the very elderly, but those de-escalation strategies should be used after reasonable case evaluation only. For irradiation of lymphatic tissue, IMRT should preferably be used and is the standard of care, especially when extended para-aortic field RT is considered to be included into the target volume [45].

Data reliability of screening scores shows a promising correlation for the identification of frailty in heterogeneous cancer patient cohorts [46,47]. However, these data are sparse in the patient population that we assessed in the present study, that is, very elderly patients treated with radiotherapy. Fiorica et al. [35] have established an ACE-27 index to be significantly associated with OS for 53 EC patients of $\geq 75$ years. Prominently, in our results, the G8 screening score was significantly predictive for OS. A comprehensive view of the geriatric patient can be ascertained using a multidimensional assessment that not only evaluates the patient's physical health with respect to medical comorbidities, but also assesses psychosocial status and functional abilities. A subjective evaluation can be very time-consuming to the busy provider, thus screening scores including the G8 seem to be a feasible and standardized approach which can achieve optimal results.

Indeed, special emphasis must be placed on reduction of treatment-related toxicity and preservation of quality of life in the elderly population. However, we advocate for strong consideration of curative treatment in this patient population which clearly has demonstrated negative oncologic results when treatment is deferred given excessive concern regarding patient age. Despite patients in our cohort all being age 80 years or older, overall clinical outcomes were quite good and relatively in line with their younger peers. Ultimately, multidisciplinary supportive care from a holistic standpoint is critical to optimize clinical outcomes in these patients $[48,49]$. In this context, future research should not rigorously exclude this underrepresented, growing subgroup, but has to redefine clinical endpoints and maybe even toxicity classifications. Further focus on studies dedicated to the very elderly is mandatory to provide more evidence for clinicians, taking into consideration that a more age-adjusted treatment approach and assessment of quality of life, changes the way how cancer therapy is implemented in clinical routine.

Major limitations of the present study include the retrospective nature of the review, which led to exclusion of patients with incomplete data for G8 rating. Subgroup analyses must therefore be interpreted cautiously and further prospective contributions to the sparse literature are greatly needed. An additional limitation is the short follow-up, albeit a consequence of the patients' age and medical comorbidities; long-term follow-up can thus be challenging, especially scheduling outpatient follow-up appointments. Nevertheless, our cohort is one of the larger single-centers reporting outcomes and acute toxicities for a homogenous patient cohort of very elderly women age $\geq 80$ years diagnosed with EC and treated adjuvant with radiotherapy with curative intent. 


\section{Conclusions}

Postoperative radiotherapy in elderly patients aged 80 years or older diagnosed with EC achieves excellent LC and OS with minimal high-grade toxicity. However, distant failure with longer follow-up remains high due to the lack of safe systemic therapy options. The geriatric G8 screening score was highly applicable for OS prognostic evaluation in our cohort. We strongly advocate for multidisciplinary assessment of the individualized oncogeriatric patient to provide safe curative postoperative treatment for those diagnosed with EC.

Author Contributions: E.M.: data curation, statistical analysis, investigation, validation, methodology, visualization, writing—original draft, writing—review, project administration, editing; K.L., L.K., E.S., J.W.L., J.D., J.H.-R.: writing—review, revision, editing; J.H.-R.: project administration, supervision. All authors have read and agreed to the published version of the manuscript.

Funding: For the publication fee we acknowledge financial support by Deutsche Forschungsgemeinschaft within the funding programme "Open Access Publikationskosten" as well as by Heidelberg University.

Institutional Review Board Statement: The study was conducted according to the guidelines of the Declaration of Helsinki, and approved by the Local Ethics Committee of the University Hospital Heidelberg (S-453/2021).

Informed Consent Statement: Individual informed consent from all subjects involved in the study was not necessary due to the Local Ethics Committee Approval.

Data Availability Statement: The data presented in this study were obtained from local databases of the Cancer Registry of the National Center for Tumor Diseases (NCT). The data are not publicly available due to Local Ethics Committee rules.

Acknowledgments: The authors thank Andreas Kudak from the Cancer Registry of the National Center for Tumor Diseases (NCT) for providing patient data.

Conflicts of Interest: The authors declare no conflict of interest. Outside the submitted work: E.M. received speaker fees from Elekta outside the submitted work. L.K. received speaker fees and travel reimbursement from Accuray International Sàrl., and NovoCure outside the submitted work. J.D. received grants from Accuray International Sàrl, Merck Serono GmbH, CRI-The Clinical Research Institute $\mathrm{GmbH}$, View Ray Inc., Accuray Incorporated, RaySearch Laboratories AB, Vision RT limited, Astellas Pharma GmbH, Astra Zeneca GmbH, Solution Akademie GmbH, Ergomed PLC Surrey Research Park, Siemens Healthcare GmbH, Quintiles GmbH, NovoCure, Pharmaceutecal Research Associates $\mathrm{GmbH}$, Boehringer Ingelheim Pharma GmbH Co., PTW-Freiburg Dr. Pychlau GmbH, Nanobiotix A.A. and IntraOP Medical outside the submitted work. JHR received speaker fees and travel reimbursement from ViewRay Inc., travel reimbursement from IntraOP Medical and Elekta Instrument $\mathrm{AB}$, a grant from IntraOP Medical outside the submitted work.

\section{References}

1. Henley, S.J.; Ward, E.M.; Scott, S.; Ma, J.; Anderson, R.N.; Firth, A.U.; Thomas, C.C.; Islami, F.; Weir, H.K.; Lewis, D.R.; et al. Annual report to the nation on the status of cancer, part I: National cancer statistics. Cancer 2020, 126, 2225-2249. [CrossRef]

2. Robert Koch Institut. Krebsregisterdaten (ZfKD) des Robert Koch-Instituts (RKI) und der Gesellschaft der Epidemiologischen Krebsregister in Deutschland: Krebs in Deutschland für 2013/2014; RKI: Berlin, Germany, 2017.

3. Onstad, M.A.; Schmandt, R.E.; Lu, K.H. Addressing the Role of Obesity in Endometrial Cancer Risk, Prevention, and Treatment. J. Clin. Oncol. 2016, 34, 4225-4230. [CrossRef]

4. $\quad$ Pike, M.C.; Peters, R.K.; Cozen, W.; Probst-Hensch, N.M.; Felix, J.C.; Wan, P.C.; Mack, T.M. Estrogen-progestin replacement therapy and endometrial cancer. J. Natl. Cancer Inst. 1997, 89, 1110-1116. [CrossRef]

5. Zeleniuch-Jacquotte, A.; Akhmedkhanov, A.; Kato, I.; Koenig, K.L.; Shore, R.E.; Kim, M.Y.; Levitz, M.; Mittal, K.R.; Raju, U.; Banerjee, S.; et al. Postmenopausal endogenous oestrogens and risk of endometrial cancer: Results of a prospective study. Br. J. Cancer 2001, 84, 975-981. [CrossRef]

6. American College of Obstetricians and Gynecologists. ACOG Committee Opinion No. 426: The role of transvaginal ultrasonography in the evaluation of postmenopausal bleeding. Obstet. Gynecol. 2009, 113, 462-464. [CrossRef]

7. Clarke, M.A.; Long, B.J.; Del Mar Morillo, A.; Arbyn, M.; Bakkum-Gamez, J.N.; Wentzensen, N. Association of Endometrial Cancer Risk with Postmenopausal Bleeding in Women: A Systematic Review and Meta-analysis. JAMA Intern. Med. 2018, 178, 1210-1222. [CrossRef] 
8. Bokhman, J.V. Two pathogenetic types of endometrial carcinoma. Gynecol. Oncol. 1983, 15, 10-17. [CrossRef]

9. Murali, R.; Davidson, B.; Fadare, O.; Carlson, J.A.; Crum, C.P.; Gilks, C.B.; Irving, J.A.; Malpica, A.; Matias-Guiu, X.; McCluggage, W.G.; et al. High-grade Endometrial Carcinomas: Morphologic and Immunohistochemical Features, Diagnostic Challenges and Recommendations. Int. J. Gynecol. Pathol. 2019, 38 (Suppl. 1), S40-S63. [CrossRef]

10. Lax, S.F. Molecular genetic pathways in various types of endometrial carcinoma: From a phenotypical to a molecular-based classification. Virchows Arch. 2004, 444, 213-223. [CrossRef]

11. FIGO; Amant, F.; Mirza, M.R.; Koskas, M.; Creutzberg, C.L. Cancer of the corpus uteri. Int. J. Gynaecol. Obstet. 2018, 143 (Suppl. 2), 37-50. [CrossRef]

12. Frost, J.A.; Webster, K.E.; Bryant, A. Lymphadenectomy for the management of endometrial cancer. Cochrane Database Syst. Rev. 2015, 2015, CD007585.

13. Holloway, R.W.; Abu-Rustum, N.R.; Backes, F.J.; Boggess, J.F.; Gotlieb, W.H.; Jeffrey Lowery, W.; Rossi, E.C.; Tanner, E.J.; Wolsky, R.J. Sentinel lymph node mapping and staging in endometrial cancer: A Society of Gynecologic Oncology literature review with consensus recommendations. Gynecol. Oncol. 2017, 146, 405-415. [CrossRef]

14. Levine, D.; The Cancer Genome Atlas Research Network. Integrated genomic characterization of endometrial carcinoma. Nature 2013, 497, 67-73, Erratum in 2013, 500, 242. [CrossRef] [PubMed]

15. Colombo, N.; Creutzberg, C.; Amant, F.; Bosse, T.; González-Martín, A.; Ledermann, J.; Marth, C.; Nout, R.; Querleu, D.; Mirza, M.R.; et al. ESMO-ESGO-ESTRO Consensus Conference on Endometrial Cancer: Diagnosis, Treatment and Follow-up. Int. J. Gynecol. Cancer 2016, 26, 2-30. [CrossRef]

16. Creutzberg, C.L.; Nout, R.A.; Lybeert, M.L.; Wárlám-Rodenhuis, C.C.; Jobsen, J.J.; Mens, J.W.; Lutgens, L.C.; Pras, E.; van de Poll-Franse, L.V.; PORTEC Study Group; et al. Fifteen-year radiotherapy outcomes of the randomized PORTEC-1 trial for endometrial carcinoma. Int. J. Radiat Oncol. Biol. Phys. 2011, 81, e631-e638. [CrossRef]

17. Nout, R.A.; Smit, V.T.; Putter, H.; Jürgenliemk-Schulz, I.M.; Jobsen, J.J.; Lutgens, L.C.; van der Steen-Banasik, E.M.; Mens, J.W.; Slot, A.; Kroese, M.C.; et al. Vaginal brachytherapy versus pelvic external beam radiotherapy for patients with endometrial cancer of high-intermediate risk (PORTEC-2): An open-label, non-inferiority, randomised trial. Lancet 2010, 375, 816-823. [CrossRef]

18. Poulsen, H.; Jacobsen, M.; Bertelsen, K.; Andersen, J.; Ahrons, S.; Bock, J.; Bostofte, E.; Engelholm, S.; Hølund, B.; Jakobsen, A.; et al. Adjuvant radiation therapy is not necessary in the management of endometrial carcinoma stage I, low risk cases. Int. J. Gynecol. Cancer 1996, 6, 38-43. [CrossRef]

19. Keys, H.M.; Roberts, J.A.; Brunetto, V.L.; Zaino, R.J.; Spirtos, N.M.; Bloss, J.D.; Pearlman, A.; Maiman, M.A.; Bell, J.G.; Gynecologic Oncology Group. A phase III trial of surgery with or without adjunctive external pelvic radiation therapy in intermediate risk endometrial adenocarcinoma: A Gynecologic Oncology Group study. Gynecol. Oncol. 2004, 92, 744-751, Erratum in 2004, 94, 241-242. [CrossRef] [PubMed]

20. Bourgin, C.; Saidani, M.; Poupon, C.; Cauchois, A.; Foucher, F.; Leveque, J.; Lavoue, V. Endometrial cancer in elderly women: Which disease, which surgical management? A systematic review of the literature. Eur. J. Surg. Oncol. 2016, 42, 166-175. [CrossRef]

21. Duska, L.; Shahrokni, A.; Powell, M. Treatment of Older Women with Endometrial Cancer: Improving Outcomes with Personalized Care. Am. Soc. Clin. Oncol. Educ. Book 2016, 35, 164-174. [CrossRef] [PubMed]

22. Benito, V.; Lubrano, A.; Andújar, M.; Mori, M.; Federico, M. Management of endometrial cancer in patients aged 80 years and older: Identifying patients who may benefit from a curative treatment. Eur. J. Obstet. Gynecol. Reprod. Biol. 2019, $242,36-42$. [CrossRef] [PubMed]

23. Kallogjeri, D.; Gaynor, S.M.; Piccirillo, M.L.; Jean, R.A.; Spitznagel, E.L., Jr.; Piccirillo, J.F. Comparison of comorbidity collection methods. J. Am. Coll Surg. 2014, 219, 245-255. [CrossRef]

24. Charlson, M.; Szatrowski, T.P.; Peterson, J.; Gold, J. Validation of a combined comorbidity index. J. Clin. Epidemiol. 1994, 47, 1245-1251. [CrossRef]

25. Bellera, C.A.; Rainfray, M.; Mathoulin-Pélissier, S.; Mertens, C.; Delva, F.; Fonck, M.; Soubeyran, P.L. Screening older cancer patients: First evaluation of the G-8 geriatric screening tool. Ann. Oncol. 2012, 23, 2166-2172. [CrossRef] [PubMed]

26. Racin, A.; Raimond, E.; Bendifallah, S.; Nyangoh Timoh, K.; Ouldamer, L.; Canlorbe, G.; Hudry, N.; Coutant, C.; Graesslin, O.; Touboul, C.; et al. Lymphadenectomy in elderly patients with high-intermediate-risk, high-risk or advanced endometrial cancer: Time to move from personalized cancer medicine to personalized patient medicine! Eur. J. Surg. Oncol. 2019, 45, 1388-1395. [CrossRef]

27. Small, W., Jr.; Mell, L.K.; Anderson, P.; Creutzberg, C.; De Los Santos, J.; Gaffney, D.; Jhingran, A.; Portelance, L.; Schefter, T.; Iyer, R.; et al. Consensus guidelines for delineation of clinical target volume for intensity-modulated pelvic radiotherapy in postoperative treatment of endometrial and cervical cancer. Int. J. Radiat. Oncol. Biol. Phys. 2008, 71, 428-434. [CrossRef]

28. Small, W., Jr.; Bosch, W.R.; Harkenrider, M.M.; Strauss, J.B.; Abu-Rustum, N.; Albuquerque, K.V.; Beriwal, S.; Creutzberg, C.L.; Eifel, P.J.; Erickson, B.A.; et al. NRG Oncology/RTOG Consensus Guidelines for Delineation of Clinical Target Volume for Intensity Modulated Pelvic Radiation Therapy in Postoperative Treatment of Endometrial and Cervical Cancer: An Update. Int. J. Radiat. Oncol. Biol. Phys. 2021, 109, 413-424. [CrossRef]

29. Bentzen, S.M.; Constine, L.S.; Deasy, J.O.; Eisbruch, A.; Jackson, A.; Marks, L.B.; Ten Haken, R.K.; Yorke, E.D. Quantitative Analyses of Normal Tissue Effects in the Clinic (QUANTEC): An introduction to the scientific issues. Int. J. Radiat. Oncol. Biol. Phys. 2010, 76, S3-S9. [CrossRef] [PubMed] 
30. Small, W., Jr.; Beriwal, S.; Demanes, D.J.; Dusenbery, K.E.; Eifel, P.; Erickson, B.; Jones, E.; Rownd, J.J.; De Los Santos, J.F.; Viswanathan, A.N.; et al. American Brachytherapy Society consensus guidelines for adjuvant vaginal cuff brachytherapy after hysterectomy. Brachytherapy 2012, 11, 58-67. [CrossRef]

31. Kenis, C.; Bron, D.; Libert, Y.; Decoster, L.; Van Puyvelde, K.; Scalliet, P.; Cornette, P.; Pepersack, T.; Luce, S.; Langenaeken, C.; et al. Relevance of a systematic geriatric screening and assessment in older patients with cancer: Results of a prospective multicentric study. Ann. Oncol. 2013, 24, 1306-1312. [CrossRef]

32. Scher, K.S.; Hurria, A. Under-representation of older adults in cancer registration trials: Known problem, little progress. J. Clin. Oncol. 2012, 30, 2036-2038. [CrossRef]

33. Zaino, R.J.; Kurman, R.J.; Diana, K.L.; Morrow, C.P. Pathologic models to predict outcome for women with endometrial adenocarcinoma: The importance of the distinction between surgical stage and clinical stage-A Gynecologic Oncology Group study. Cancer 1996, 77, 1115-1121, Erratum in 1997, 79, 422. [CrossRef]

34. Alektiar, K.M.; Venkatraman, E.; Abu-Rustum, N.; Barakat, R.R. Is endometrial carcinoma intrinsically more aggressive in elderly patients? Cancer 2003, 98, 2368-2377. [CrossRef]

35. Fiorica, F.; Zini, G.; Cartei, F.; Candela, M.V.; Pascale, G.; Berretta, M.; Lleshi, A.; Falco Abramo, M.R.; Del Pup, L.; Fisichella, R.; et al. Postoperative radiotherapy and comorbidity assessment in older and oldest elderly endometrial cancer patients: A retrospective analysis. WCRJ 2014, 1, e55.

36. Truong, P.T.; Kader, H.A.; Lacy, B.; Lesperance, M.; MacNeil, M.V.; Berthelet, E.; McMurtrie, E.; Alexander, S. The effects of age and comorbidity on treatment and outcomes in women with endometrial cancer. Am. J. Clin. Oncol. 2005, 28, 157-164. [CrossRef] [PubMed]

37. Montemaggi, P.; Guerrieri, P. Brachytherapy in the elderly. Crit. Rev. Oncol. Hematol. 2001, 37, 159-167. [CrossRef]

38. Hurria, A.; Togawa, K.; Mohile, S.G.; Owusu, C.; Klepin, H.D.; Gross, C.P.; Lichtman, S.M.; Gajra, A.; Bhatia, S.; Katheria, V.; et al. Predicting chemotherapy toxicity in older adults with cancer: A prospective multicenter study. J. Clin. Oncol. 2011, 29, 3457-3465. [CrossRef]

39. Manzano, J.G.; Luo, R.; Elting, L.S.; George, M.; Suarez-Almazor, M.E. Patterns and predictors of unplanned hospitalization in a population-based cohort of elderly patients with GI cancer. J. Clin. Oncol. 2014, 32, 3527-3533. [CrossRef] [PubMed]

40. Smith, A.W.; Reeve, B.B.; Bellizzi, K.M.; Harlan, L.C.; Klabunde, C.N.; Amsellem, M.; Bierman, A.S.; Hays, R.D. Cancer, comorbidities, and health-related quality of life of older adults. Health Care Financ. Rev. 2008, 29, 41-56. [PubMed]

41. Giacalone, A.; Quitadamo, D.; Zanet, E.; Berretta, M.; Spina, M.; Tirelli, U. Cancer-related fatigue in the elderly. Support. Care Cancer 2013, 21, 2899-2911. [CrossRef]

42. Mundt, A.J.; Lujan, A.E.; Rotmensch, J.; Waggoner, S.E.; Yamada, S.D.; Fleming, G.; Roeske, J.C. Intensity-modulated whole pelvic radiotherapy in women with gynecologic malignancies. Int. J. Radiat. Oncol. Biol. Phys. 2002, 52, 1330-1337. [CrossRef]

43. Wortman, B.G.; Post, C.C.B.; Powell, M.E.; Khaw, P.; Fyles, A.; D’Amico, R.; Haie-Meder, C.; Jürgenliemk-Schulz, I.M.; McCormack, M.; Do, V.; et al. Radiation Therapy Techniques and Treatment-Related Toxicity in the PORTEC-3 Trial: Comparison of 3-Dimensional Conformal Radiation Therapy Versus Intensity-Modulated Radiation Therapy. Int. J. Radiat. Oncol. Biol. Phys. 2021. [CrossRef] [PubMed]

44. de Jong, R.A.; Pras, E.; Boezen, H.M.; van der Zee, A.G.; Mourits, M.J.; Arts, H.J.; Aalders, J.G.; Slot, A.; Timmer, P.R.; Hollema, H.; et al. Less gastrointestinal toxicity after adjuvant radiotherapy on a small pelvic field compared to a standard pelvic field in patients with endometrial carcinoma. Int. J. Gynecol. Cancer 2012, 22, 1177-1186. [CrossRef]

45. Poorvu, P.D.; Sadow, C.A.; Townamchai, K.; Damato, A.L.; Viswanathan, A.N. Duodenal and other gastrointestinal toxicity in cervical and endometrial cancer treated with extended-field intensity modulated radiation therapy to paraaortic lymph nodes. Int. J. Radiat. Oncol. Biol. Phys. 2013, 85, 1262-1268. [CrossRef]

46. Hamaker, M.E.; Jonker, J.M.; de Rooij, S.E.; Vos, A.G.; Smorenburg, C.H.; van Munster, B.C. Frailty screening methods for predicting outcome of a comprehensive geriatric assessment in elderly patients with cancer: A systematic review. Lancet Oncol. 2012, 13, e437-e444. [CrossRef]

47. Baitar, A.; Van Fraeyenhove, F.; Vandebroek, A.; De Droogh, E.; Galdermans, D.; Mebis, J.; Schrijvers, D. Evaluation of the Groningen Frailty Indicator and the G8 questionnaire as screening tools for frailty in older patients with cancer. J. Geriatr. Oncol. 2013, 4, 32-38. [CrossRef]

48. Kaufmann, A.; Schmidt, H.; Ostheimer, C.; Ullrich, J.; Landenberger, M.; Vordermark, D. Quality of life in very elderly radiotherapy patients: A prospective pilot study using the EORTC QLQ-ELD14 module. Support. Care Cancer 2015, 23, 1883-1892. [CrossRef] [PubMed]

49. Huguenin, P.; Glanzmann, C.; Lütolf, U.M. Acute toxicity of curative radiotherapy in elderly patients. Strahlenther. Onkol. 1996, 172, 658-663. 